\title{
Decision on conducting HCV Immunoblot and HCV Viral Load Tests Dependent upon the Result of the Screening Tests
}

\section{Francisco Bischoff, Maria do Carmo Torres Silva Duarte Teixeira Koch and Fernando Manuel Ferreira Araújo}

\author{
Centro Hospitalar de São João, E.P.E., Alameda Prof. Hernâni Monteiro, Portugal
}

Correspondence should be addressed to: Francisco Bischoff; franzbischoff@gmail.com

Received 24 January 2013; Accepted 13 March 2013; Published 31 May 2013

Academic Editor: Vicente Carreño

Copyright (C) 2013 Francisco Bischoff, Maria do Carmo Torres Silva Duarte Teixeira Koch and Fernando Manuel Ferreira Araújo. Distributed under Creative Commons CC-BY 3.0

\begin{abstract}
Testing for the presence of antibody to hepatitis $\mathrm{C}$ virus (anti-HCV) is recommended for initially identifying persons with HCV infection. According to the CDC guidelines it is appropriate to use a signal-to-cut-off value (S/CO) to limit the number of samples that needs supplemental testing. Moreover, the use of quantitative PCR assays for HCV RNA testing is fundamental for the assessment of chronic hepatitis C. The purpose of this study is to determine a specific value for a serological test for anti-HCV with a Positive Predictive Value (PPV) of $95 \%$ on positive HCV Immunoblot, and also determine a cut-off value for performing a clinically relevant HCV PCR. Were observed 415 individuals identified de novo as anti-HCV reactive, between 2009 and 2011. We estimate that a S/CO of 6.0 has a PPV of $99.83 \%$ being positive Immunoblot assay and that $99.49 \%$ of the samples with a S/CO $\leq 6.0$ will have no detectable virus on PCR. Based on these results we propose a new algorithm for evaluation persons identified de novo as anti-HCV reactive: Immunoblot assay needs to be performed only for samples with a S/CO $\leq 6.0$ and HCV PCR will be performed for persons with a $\mathrm{S} / \mathrm{CO}>6.0$. Using these criteria it would be possible to save $€$ $9,000 /$ year with acceptable clinical accuracy. This algorithm does not apply to rare cases of suspected acute HCV infection or suspicion of HCV infection in immunocompromised patients; for these cases we maintain the current approach of NAT testing for laboratory diagnosis of HCV infection.
\end{abstract}

Keywords: HCV, Inno-lia, PCR, Immunoblot.

\section{Introduction}

Diagnosis of HCV infection is based on detection of antibodies to hepatitis $\mathrm{C}$ (antiHCV) antibodies by immunoassay and detection of HCV RNA by a sensitive molecular method (lower limit of detection $<50 \mathrm{IU} / \mathrm{ml}$ ), ideally a real-time PCR assay.
An appropriate use of the available laboratory assays is crucial for an accurate and efficient diagnosis of HCV infection because there are important medical and social implications for persons designated as having HCV infection.

Testing for the presence of antibodies to hepatitis $\mathrm{C}$ virus (anti-HCV) is recommended

Cite this Article as: Francisco Bischoff, Maria do Carmo Torres Silva Duarte Teixeira Koch and Fernando Manuel Ferreira Araújo (2013), "Decision on conducting HCV Immunoblot and HCV Viral Load Tests Dependent upon the Result of the Screening Tests," Journal of Virology \& Microbiology, Vol. 2013 (2013), Article ID 332501, DOI: 10.5171/2013. 332501 
for initially identifying persons with HCV infection and should include the use of an anti-HCV screening test and a more specific supplemental assay. According to CDC guidelines it is appropriate to use a signal-tocut-off value (S/CO) to limit the number of samples that needs supplemental testing. Moreover, the quantitative test (viral load) of polymerase chain reaction for HCV RNA (PCR HCV) is fundamental for the assessment of chronic hepatitis $\mathrm{C}$, and due to its high cost it must be used judiciously.

With the purpose of reducing costs by eliminating unnecessary lab tests, the authors intended to determine a specific value of anti-HCV ratio (S/CO) in screening tests that can predict with a Positive Predictive Value (PPV) of $95 \%$ a true-positive result for $\mathrm{HCV}$, and also determine a cut-off value for performing a clinically relevant PCR HCV.

\section{Materials and Methods}

The analysis reported here is based on files from our laboratory, at Centro Hospitalar São João, containing information about patients, results of HCV serologic screening and supplemental test results.

It includes all patients identified de novo as anti-HCV reactive, from January 1, 2009, through December 31, 2011.

In our lab, testing for HCV infection is performed for clinical diagnosis of patients with signs or symptoms of liver disease and for screening asymptomatic persons to identify HCV-infected persons who should receive counselling and medical evaluation.

Anti-HCV testing includes initial screening with Architect Anti-HCV assay on the Architect i2000SR system (Abbott Laboratories, Abbott Park, IL). This assay is a two-step immunoassay for the qualitative detection of hepatitis C antibody (anti-HCV) in human serum or plasma using chemiluminescent assay (CMIA) technology.

The resulting chemiluminescent reaction is measured as relative light units (RLUs). A relationship exists between the amount of anti-HCV in the sample and the RLUs detected by the Architect i optical system. For each sample the Architect Anti-HCV assay protocol calculates a result based on the ratio of the sample RLU (S) to the cutoff RLU (CO).

Samples showing repeat reactive results ( $\mathrm{S} / \mathrm{CO} \geq 0.9$ ) are tested with the Immunoblot assay INNO-LIA ${ }^{\mathrm{TM}}$ HCV Score (Innogenetics) on Auto-LiPA equipment. This assay utilizes well-defined antigens derived from HCV immunodominant proteins from the core region, the E2 hypervariable region (HVR), the NS3 helicase region and the NS4A, NS4B and NS5A regions. The antigens used are either recombinant proteins or synthetic peptides, highly purified, and fixed on a nylon membrane. In addition the strip includes four control lines: a streptavidin control line, weak and medium positive control lines (human IgG), and a strong positive control line (anti-human IgG) which is also the sample addition control line. The interpretation of results is performed according to the manufacturer's instructions.

A negative Immunoblot result is interpreted as anti-HCV negative. Nucleic acid testing (NAT) is not performed.

NAT testing, for the detection of HCV RNA, is carried out, in the cases of INNO-LIA positive or indeterminate.

In both cases, after patient's physician request, the detection of HCV RNA is performed with a new fresh specimen, using a real time PCR assay (PCR COBAS Ampliprep/COBAS TAqMan-HCV Quantitative Test v2.0-Roche(C), with a diagnostic sensivity and linearity from $\geq 15$ $\mathrm{UI} / \mathrm{mL}$.

Statistical study was based on analysis of the one-tailed distribution curves, aiming to find a ratio $(\mathrm{S} / \mathrm{CO})$ where the area under the curve was $>95.00 \%$ for both parameters.

\section{Results}

During the three-year study period, 415 patients were identified de novo as anti-HCV reactive $(\mathrm{S} / \mathrm{CO} \geq 0.9$ ).

After performing INNO-LIA test, 266 patients $(64.1 \%)$ were classified as anti-HCV positive, 114 patients $(27.5 \%)$ as anti-HCV Negative and 35 patients $(8.4 \%)$ as Indeterminate.

None of patients classified as anti-HCV Negative had been tested for HCV RNA. The 
patients classified as anti-HCV Indeterminate had been tested for HCV RNA and all were RNA PCR Not Detected.

Out of 266 patients with INNO-LIA positive result, 149 (56\%) had been tested for HCV RNA.

For statistical purpose, INNO-LIA test results have been divided into two groups: Positive (266 patients) and Negative/Indeterminate (149 patients). For each group we calculate the average, standard deviation and standard error of Architect anti-HCV screening test result $(\mathrm{S} / \mathrm{CO})$ and the data was analyzed to determine a specific ratio (S/CO) that could predict with a PPV greater than $95 \%$ a truepositive anti-HCV. Table 1.

Table 1: Results of INNO-LIATM in 415 Samples with Anti-HCV (S/CO) $\geq 0,9$

\begin{tabular}{|l|l|l|}
\hline & INNO-LIA $^{\text {TM Negative/Indeterminate }}$ & INNO-LIATM Positive \\
\hline $\mathbf{N}$ & $149(115 / 34)$ & 266 \\
\hline Min $^{\mathbf{a}}$ & 1.0 & 1.1 \\
\hline Max $^{\mathbf{a}}$ & 7.3 & 17.6 \\
\hline Average $^{\mathbf{a}}$ & $2.10(2.22 / 1.70)$ & 11.49 \\
\hline Standard deviationa $^{\mathbf{a}}$ error & $1.23(1.35 / 0.56)$ & 3.46 \\
\hline $\begin{array}{l}\text { Standard } \\
(\mathbf{9 5 \%})^{\mathbf{a}}\end{array}$ & $0.20(0.25 / 0.19)$ & 0.42 \\
\hline
\end{tabular}

a Ratio (S/CO)

Also for statistical purpose, NAT test results belonging to 149 patients with INNO-LIA positive have been divided into two groups: RNA Detected (88) and RNA Not Detected (61). For each group we calculate the average, standard deviation and standard error of Architect anti-HCV screening test result $(\mathrm{S} / \mathrm{CO})$, and the data were analyzed to calculate a ratio $(\mathrm{S} / \mathrm{CO})$ below which we could predict (with a probability greater than 95\%) a RNA HCV Not Detected. Table 2.

Table 2: Results of PCR HCV in 149 INNO-LIA Positive Samples

\begin{tabular}{|l|l|l|}
\hline & PCR HCV "Not Detected" & V “Detected" \\
\hline $\mathbf{N}$ & 61 & 88 \\
\hline Min $^{\mathbf{a}}$ & 1.0 & 6.0 \\
\hline Max $^{\mathbf{a}}$ & 16.0 & 16.6 \\
\hline Average $^{\mathbf{a}}$ & 6.59 & 12.27 \\
\hline Standard deviationa $^{\mathbf{a}}$ & 5.08 & 2.21 \\
\hline $\begin{array}{l}\text { Standard error } \\
(\mathbf{9 5 \% )}\end{array}$ & 1.29 & 0.47 \\
\hline
\end{tabular}

a Ratio (S/CO)

In Figure 1 we can see the distribution of INNO-LIA and PCR HCV test results. When we analyze the data related to the INNO-LIA test, we can see that for a value (S/CO) of 5.8 (95 percent confidence interval: $5.6-6.0$ ) in the screening test, $99.83 \%$ of results with value greater than or equal to 5.8 will be INNO-LIA positive and $0.17 \%$ will be INNO-LIA Negative. Regarding to PCR HCV, we can see that for a value (S/CO) of 6.47 (CI 95\%: 6.0 6.93 ) in the screening test, $99.49 \%$ of results with value lower than or equal to 6.47 will be PCR HCV "Not Detected" and 0.51\% will be PCR HCV positive. 


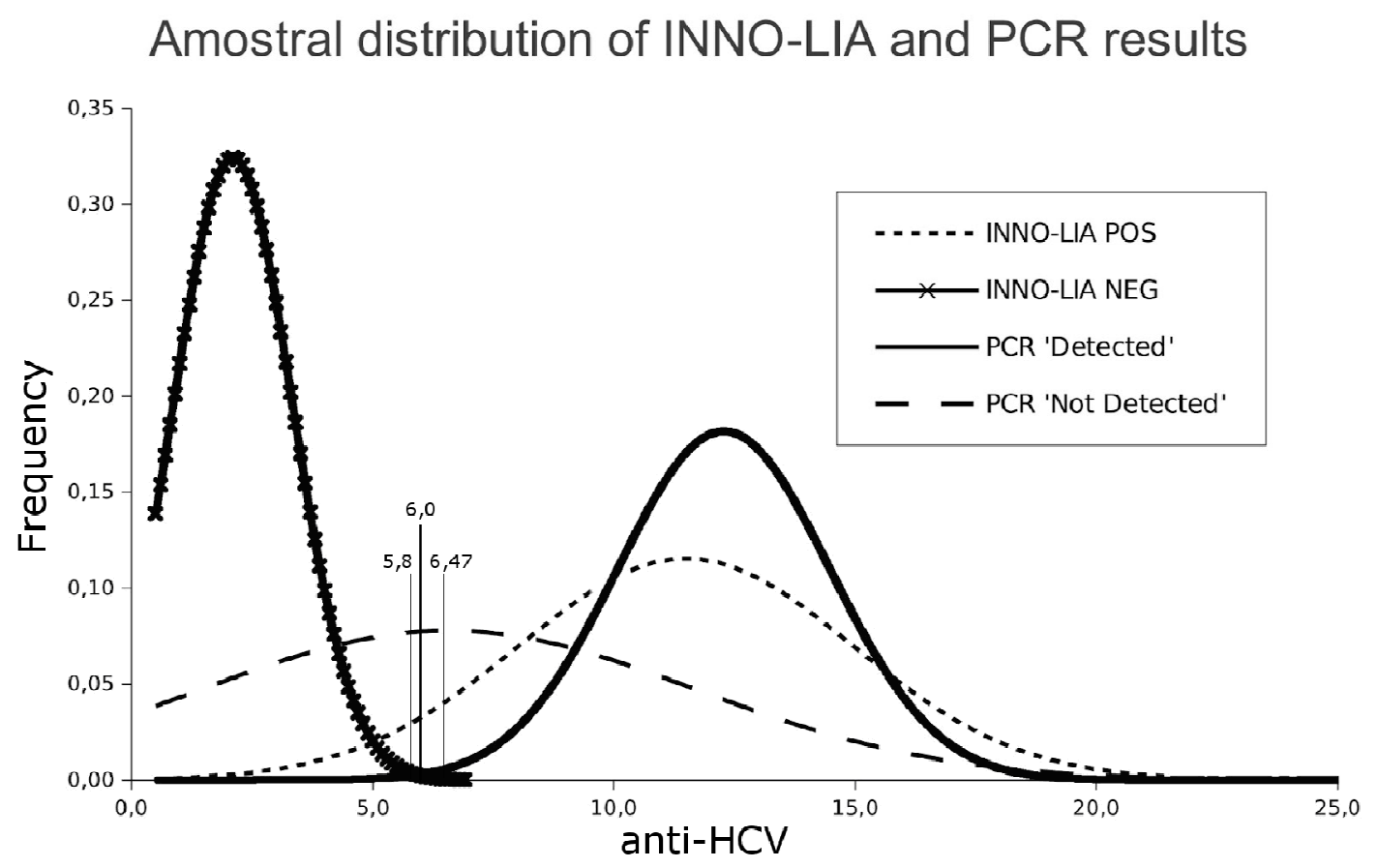

Figure 1: Amostral Distribution of INNO-LIA and PCR Results, According to the Values of (S/CO) Obtained from the Screening Test

According to these results and to facilitate the creation of a laboratorial algorithm, we use the common value $(\mathrm{S} / \mathrm{CO}=6)$ to both confidence intervals of the estimated cut-offs, for INNO-LIATM and PCR HCV, as an overall cut-off, proposing the algorithm displayed in Figure 2.

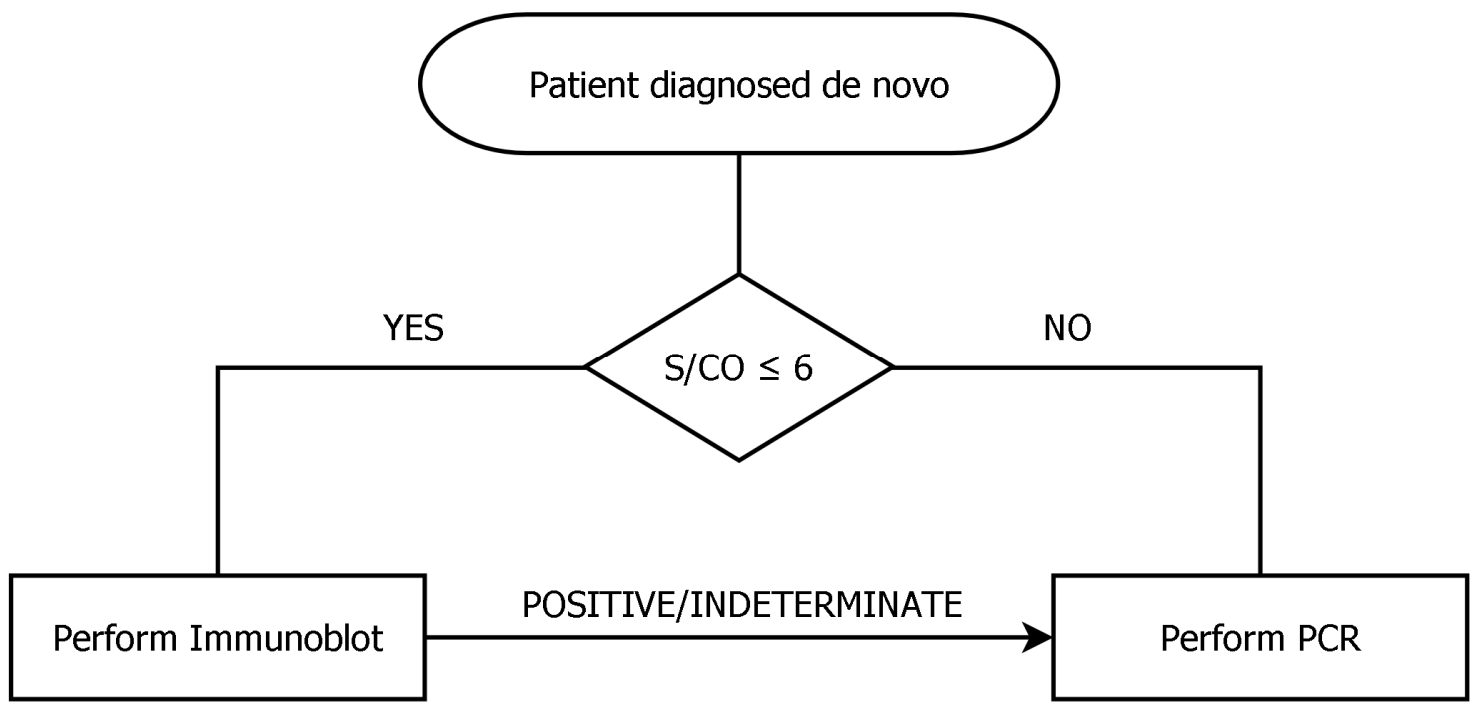

Figure 2: Laboratorial Algorithm for Guidance in Performing Analysis 


\section{Discussion}

According to the European Association for the Study of the Liver (EASL), the diagnosis of chronic hepatitis $\mathrm{C}$ is based on the detection of $\mathrm{HCV}$ infection (positive anti-HCV antibodies and HCV RNA) in a patient with signs of chronic hepatitis. Rarely, in profoundly immunosuppressed patients, anti-HCV antibodies are not detected and HCV RNA is present alone.

Some studies showed that it is appropriate to use a signal-to-cut-off value (S/CO) to limit the number of samples that needs supplemental testing. However, given the existence of different equipment and methodologies, this value cannot be generalized and it is highly recommended that each laboratory/hospital calculate this value depending on the equipment and methodologies available to them. As a positive anti-HCV result may indicate past infection or active infection, the NAT testing is important to differentiate these two situations.

With this study the authors intended to calculate two cut-off values (S/CO) for the screening test anti-HCV (Architect AbbottC): A value above which the assay predicts a positive Immunoblot result, over $95 \%$ of the time, and a value below which, it predicts a negative PCR by more than $95 \%$ of the time, in the study population.

Using data belonging to patients identified de novo as anti-HCV reactive, from 2009 to 2011, two cut-off values were obtained: $\mathbf{5 . 8}$ (CI 95\%: 5.6 - 6.0), indicating that results above or equal to 5.8 are true positives in Immunoblot test (with a probability greater than 99\%) and 6.47 (CI 95\%: 6.0 - 6.93), indicating that the results below 6.47 have undetectable viral load (with a probability greater than 99\%). Using the common value to both confidence intervals, we defined a global cut-off value, to build a simple algorithm, in patients identified de novo, for the laboratory diagnosis of HCV infection.

Our data shows that out of the 415 reactive results $(\geq 0.9), 27.5 \%$ were classified as negative, $8.4 \%$ as indeterminate and $64.1 \%$ as positive in Immunoblot test. An indeterminate result indicates that the reading pattern was inconclusive and may indicate a process of seroconversion but usually corresponds to a false reactive, particularly in low-risk populations.

In our study, the patients classified as antiHCV Indeterminate had been tested for HCV RNA and all were RNA PCR Not Detected. All PCR HCV positive cases had a prior positive test result in the Immunoblot assay.

The study population included individuals with different prevalence of HCV infection, including patients with liver disease, haemodialysis patients and healthcare workers.

The EASL states that the diagnosis of HCV infection is based on the evidence of a positive anti-HCV and PCR HCV, but does not states against the use of Immunoblotting or recommend any laboratorial algorithm. The American Association for the Study of Liver Diseases (AASLD) Practice Guidelines states that nowadays the Immunoblotting has no role for high S/CO ratios due to extremely high specificity for third generation EIA. This affirmation is consistent with our algorithm. According to the HCV Infection Testing for Diagnosis Flow Chart from CDC, there is no rule to choose between PCR HCV or Immunoblot when the screening test has a low S/CO ratio and no other test has been done. CDC has also published a cut-off value for Architect Anti-HCV screening test with a value of $\geq 5.0$ (predictive of a true positive $\geq$ 95\%), but this do not take in account a cut-off for PCR HCV. Our algorithm suggests that we should use the Immunoblot assay for low S/CO ratios, and PCR HCV for high S/CO ratios.

It should be noted that if we adopt the proposed laboratorial algorithm, a HCV RNA quantitative assay must always be done if a positive or indeterminate Immunoblot result is obtained, even below the proposed cut-off value. This is very crucial for the individuals with an anti-HCV positive test not only for HCV diagnosis but also for the evaluation of treatment.

This algorithm does not apply to rare cases of suspected acute HCV infection or suspicion of HCV infection in immunocompromised patients; for these cases we maintain the current approach of NAT testing for laboratory diagnosis of HCV infection. 
Searching for S/CO ratios among special populations, such as related with occult HCV infection, would add clinical value and further studies should be conducted.

\section{Conclusions}

The results of these study allow us to state that, in patients identified de novo in the antiHCV screening test, if the result obtained (S/CO) is $>6.0$, the probability of obtaining a INNO-LIA ${ }^{\mathrm{TM}} \mathrm{HCV}$ positive result is $>99.83 \%$, and therefore we propose that the Immunoblot test should be performed only when the S/CO of anti-HCV screening test is $\leq$ 6.0 .

The study also allow us to suggest that if the anti-HCV screening test has a S/CO of $\leq 6.0$, the probability of obtaining a result PCR HCV "Not Detected" is $\geq 99.49 \%$, and therefore we propose that PCR HCV shall be performed only when the S/CO of anti-HCV screening test is greater than 6.0 .

The financial impact related with the implementation of this new approach was estimated. During the three-year study, an average of 138 INNO-LIA ${ }^{\mathrm{TM}}$ and 50 PCR HCV tests were performed annually, on patients identified de novo. Of these, 81 INNO-LIA ${ }^{\mathrm{TM}}$ tests had results (S/CO) of $>6.0$ and 13 PCR $\mathrm{HCV}$ had results $(\mathrm{S} / \mathrm{CO})$ of $\leq 6.0$.

According to the ordinance of Official Gazette of Portugal, the cost is set at $€ 102.90 /$ sample for the INNO-LIA ${ }^{\mathrm{TM}}$ supplementary test, and $€ 66.80 /$ sample for PCR HCV, which totals $€ 17,540.20 / y e a r$, if the previous strategy is maintained. Adopting the approach proposed by this study, in immunocompetent populations, it would be possible, at best, to save $\approx € 9,203.30 /$ year, i.e. more than a half of that expenditure $(\approx 52.47 \%)$, without jeopardizing the quality of laboratory testing.

The implementation of an appropriate algorithm, based on a specific value of the screening test to determine which test to be carried out subsequently, facilitates and enhances the laboratory diagnosis in patients identified de novo, allowing a substantial reduction in costs.

\section{Limitations of the Study}

There are some limitations that need to be acknowledged and addressed regarding the present study. The first limitation concerns the target population of this research that is limited to patients with different prevalence of HCV infection, among which were patients with liver disease, haemodialysis patients and healthcare workers. Blood donors were not included. Therefore we have a heterogeneous population, however this is common in a hospital-based laboratory. Another concern is the timing, this study only analyzed persons identified de novo, and cannot be generalized for follow-up patients.

Finally, the available data is limited, since this is a retrospective study and there was no PCR available for negative Immunoblot tests.

Therefore we advise that these results should be interpreted with caution, and it is highly recommended that each laboratory/hospital calculate the cut-off value depending on the equipment and methodologies available to them.

\section{References}

AASLD. (2009, Apr). "Diagnosis, Management, and Treatment of Hepatitis C: An Update," Hepatology, 49(4) 1335-74.

CDC. (1998, Oct). 'Recommendations for Prevention and Control of Hepatitis C Virus [HCV] Infection and HCV-Related Chronic Disease,' Morbidity and Mortality Weekly Report. Recommendations and Reports, 47 (RR-19) 1-33.

CDC. (2003, Feb). Guidelines for Laboratory Testing and Result Reporting of Antibody to Hepatitis C Virus, Morbidity and Mortality Weekly Report. Recommendations and Reports, 52 (RR-3) 1-15.

CDC DVH. (2008, July). HCV Infection Testing for Diagnosis Flow Chart, [Online], [Retrieved January 14, 2013] http://www.cdc.gov/hepatitis/HCV/PDFs/hc v_flow.pdf

CDC DVH. (2008, July). "Signal-to-Cut-Off Ratios for Commercially Available Assays," [Online], [Retrieved January 14, 2013] http://www.cdc.gov/hepatitis/HCV/LabTest ing.htm

Chevaliez, S., Rodriguez, C. \& Pawlotsky, J. M. (2012, May). "New Virologic Tools for 
Management of Chronic Hepatitis B and C," Gastroenterology, 142 (6) 1303-1313.e1.

European Association for the Study of the Liver. (2011, Aug). "EASL Clinical Practice Guidelines: Management of Hepatitis C Virus Infection," Journal of Hepatology, 55 (2) 245264.

Kleinman, S., Alter, H., Busch, M. et al. (1992, Nov-Dec). "Increased Detection of Hepatitis C
Virus (HCV)-Infected Blood Donors by a Multiple-antigen HCV Enzyme Immunoassay," Transfusion, 32 (9) 805-13.

Payan, C., Raimbert, A., Fouchard-Hubert, I., Kouyoumdjian, S. \& Lunel-Fabiani, F. (2003, May-Jun). "Quantitative Antibody Analysis: Use for the Diagnosis of Hepatitis C Virus Chronic Infection," Ann Biol Clin (Paris), 61 (3) 311-317. 